\title{
Impacto microbiológico y toxicológico del cauce del rio balsas, región tierra caliente de
}

\section{Guerrero}

Microbiological and toxicological impact of the river basin, hot land region of Guerrero

\author{
Escobar Sarabia Ludybed ${ }^{1}$, Ma. Guadalupe Álvarez Díaz ${ }^{1}$, Diana Pérez de Jesús ${ }^{1}$, Francisco \\ Zavala Hernández ${ }^{1,2}$ \\ ${ }^{1}$ Academia de Biología, Instituto Tecnológico de Cd. Altamirano, Av. Pungarabato Pte. S/N Col. \\ Morelos, Cd.Altamirano, Guerrero, México. CP. 40660. Tel y Fax: (767) 6721213
}

凶Autor para correspondencia: zavalahf@yahoo.com.mx

Recibido: 15/04/2019

Aceptado: 15/05/2019

\section{RESUMEN}

En los últimos quince años se ha registrado un alto grado de deterioro en el río Balsas, México, debido a los asentamientos humanos e industriales. El objetivo de este estudio fue evaluar la condición actual del río y establecer la relación entre los parámetros de la legislación vigente y las pruebas de toxicidad y mutagenicidad. Se determinaron parámetros de campo (color, olor, temperatura, CE, pH, OD), fisicoquímicos (SST, SDT, SSe, DBO5, DQO y GyA, Ntotal, Norg, Ptotal, Porg, ortofosfatos), metales pesados (As, Cd, Cu, Cr, Cr+6, Hg, Ni, Pb, Zn, Al, Fe, Mn), microbiológicos (coliformes fecales) con base en los límites máximos permisibles (LMP) en la NOM-001-ECOL-1996 y en los CE-CCA-001/89, análisis de toxicidad (Vibrio fischeri, Daphnia magna) y mutagenicidad (prueba de Ames con microsomas/Salmonella typhimurium), en época de sequía en nueve estaciones a lo largo de $85 \mathrm{~km}$. Los valores para OD, DBO5 y DQO fueron de 2-6, 11-270 y 22-1841 mg.1-1, respectivamente. Los metales fueron detectados por debajo de los LMP en todas las estaciones, excepto en la estación $5(0,002 \mathrm{mg} \cdot 1-1 \mathrm{de} \mathrm{Hg})$ y la $8(0,13 \mathrm{mg} \cdot 1-1 \mathrm{de} \mathrm{Cr}+6)$. Los coliformes fecales rebasaron los LMP en todas las estaciones. Los valores para Vibrio fischeri, Daphnia magna se encuentran en 2-28 y 1-4UT, y para la prueba de Ames entre 5 y 63RM, indicando toxicidad y mutagenicidad desde la estación 3 a la 9. Se registró contaminación alta según la legislación vigente y un riesgo para la salud pública de acuerdo a los parámetros toxicológicos y mutagénicos.

Palabras clave: Rio Balsas, Indicadores de calidad de agua, parámetros toxicológicos.

\begin{abstract}
In the last fifteen years there has been a high degree of deterioration in the Balsas River, Mexico, due to human and industrial settlements. The objective of this study was to evaluate the current condition of the river and establish the relationship between the parameters of the current legislation and the toxicity and mutagenicity tests. Field parameters (color, odor, temperature, EC, pH, OD), physicochemical (SST, SDT, SSe, BOD5, COD and GyA, Notalus, Norg, Ptotal, Porg, orthophosphates), heavy metals (As, $\mathrm{Cd}$ ) were determined. $\mathrm{Cu}, \mathrm{Cr}, \mathrm{Cr}+6, \mathrm{Hg}, \mathrm{Ni}, \mathrm{Pb}, \mathrm{Zn}, \mathrm{Al}, \mathrm{Fe}$, $\mathrm{Mn}$ ), microbiological (fecal coliforms) based on the maximum permissible limits (LMP) in NOM001-ECOL-1996 and in CE-CCA-001/89, toxicity analysis (Vibrio fischeri, Daphnia magna) and mutagenicity (Ames test with microsomes / Salmonella typhimurium), during the dry season in nine
\end{abstract}


stations along $85 \mathrm{~km}$. The values for OD, BOD5 and COD were 2-6, 11-270 and 22-1841 $\mathrm{mg} \cdot 1-1$, respectively. The metals were detected below the LMPs in all stations, except for station $5(0.002 \mathrm{mg}$ $\cdot 1-1$ of $\mathrm{Hg})$ and $8(0.13 \mathrm{mg} \cdot 1-1$ of $\mathrm{Cr}+6)$. Fecal coliforms exceeded PML in all seasons. Values for Vibrio fischeri, Daphnia magna are found in 2-28 and 1-4UT, and for the Ames test between 5 and 63RM, indicating toxicity and mutagenicity from station 3 to 9. High contamination was recorded according to current legislation and a risk to public health according to the toxicological and mutagenic parameters.

Keywords: Rio Balsas, water quality indicators, toxicological parameters.

\section{INTRODUCCIÓN}

La problemática ambiental del río Balsas es el resultado del deterioro producido por la expansión demográfica sostenida y el desarrollo económico de los estados de Puebla y Tlaxcala, que se asientan en $2429 \mathrm{~km}^{2}$ de extensión superficial de la subcuenca del Atoyac (Figura 1). Las actividades socioeconómicas de la zona son: alimenticia, textil, química, petroquímica, automotriz, papelera, bebidas, hierro y acero, farmacéutica, tenería, metalmecánica, siderúrgica y producción agrícola (INEGI, 2004). Estas actividades producen aproximadamente 55 descargas de aguas residuales directas e indirectas, 45 industriales y 10 municipales (CONAGUA, 2007b).En los últimos 14 años, diferentes estudios han caracterizado la calidad del agua del río Atoyac y de las zonas de impacto a su alrededor, determinando las condiciones y el riesgo que presentan las descargas depositadas en él. En 1995, Méndez et al reportó que la incorporación del agua residual procedente de Tlaxcala, San Martín Texmelucan, Moyotzingo y la Ciudad de Puebla al río provocó el incremento del contenido de sales solubles, metales pesados, detergentes y grasas en la zona. Posteriormente, Méndez et al. (2000) muestrearon suelos sometidos a riego por más de 30 años con aguas del río, revelando que el agua presentaba concentraciones de metales en el orden
$\mathrm{Fe}>\mathrm{Pb}>\mathrm{Mn}>\mathrm{Cr}>\mathrm{Cd}$, detectándolos en el suelo analizado. Silva et al. (2002) analizaron la calidad del agua de la región atlixquense, que incluye el río Nexapa, afluente del Atoyac, mostrando que se excedieron LMP para parámetros como DBO5, DQO, dureza, temperatura, nitrógeno amoniacal, $\mathrm{pH}, \mathrm{SS}$, turbiedad, $\mathrm{Pb}, \mathrm{Cr}, \mathrm{Cd}$ y $\mathrm{Zn}$. Saldaña et al., (2002a), empleando pruebas de toxicidad en la evaluación de la calidad del agua del río Alseseca, que al igual que el Atoyac desemboca en la presa Manuel Ávila Camacho, encontraron un coeficiente de correlación alto entre parámetros fisicoquímicos y toxicológicos. Saldaña et al. (2002b) reportaron 522 descargas municipales e industriales en Estado de Puebla, que en su mayoría cumplió con los parámetros de la NOM-001-ECOL-1996 y resaltaron la importancia de incluir en la norma las pruebas de toxicidad con Photobacterium phosphoreum para evaluar sustancias tóxicas no detectadas por los análisis convencionales. En el estudio de viabilidad de uso acuícola de Ciprinus carpio en la Presa Manuel Ávila Camacho, Flores et al. (2004) demostraron que la concentración de metales pesados en el agua de la presa Valsequillo no rebasó el LMP para su uso en riego de cultivos, además de la ausencia de elementos tóxicos en el músculo de peces. Sin embargo, el grado de contaminación en el embalse de la presa lo hace no apto para consumo humano. Mangas et al. (2005) resaltaron la gravedad en que se 
encuentra la presa Valsequillo y discuten diversos intentos para rehabilitarla, cuyos logros han quedado al margen de las metas propuestas. Saldaña y Gómez (2006) incluyeron en su estudio un parámetro de toxicidad (Vibrio fischeri señalando que 17 de las 23 descargas analizadas no cumplen con la norma y 16 de ellas presentan toxicidad de 2 1165unidades de toxicidad (UT); sin embargo, los parámetros de toxicidad no están incluidos en la norma mexicana. CONAGUA (2007a) reportó que las aguas residuales de los ríos Atoyac y Zahuapan rebasaron en 2005 los LMP de DBO5, DQO, metales y toxicológicos, proponiendo un acuerdo para el saneamiento de la presa Valsequillo con metas en tres etapas. Sin embargo, el nivel de contaminación en la presa sigue siendo alto, lo que demuestra la necesidad de disminuir el impacto de las descargas.

En América Latina existen estudios sobre construcción de índices de calidad en humedales tropicales en la costa sur de México (Escobedo Urías et al., 2011) y en la cuenca del Río Suquia (Córdoba, Argentina) donde Pesce y Wunderlin (2000) desarrollaron un índice mínimo de exitosa aplicación. El análisis cuali-cuantitativo del agua en América Latina $y$ el Caribe tiene una particular importancia, dado que a pesar de contar con el $30 \%$ de los recursos hídricos del mundo la distribución de la población con respecto a estos recursos es irregular e inequitativa, ya que la mayor parte de las personas viven en áreas donde el agua, si existe, escasea y/o está contaminada. Por este motivo dentro de los objetivos del milenio se encuentran el suministro de agua, el saneamiento y la gestión de los recursos hídricos (PNUMA, 2003). Por lo anterior se realizó un estudio un diagnóstico físico - químico y microbiológico del agua superficial en la cuenca del rio Balsas de la Región Tierra Caliente de Guerrero, donde se realizó un diagnóstico ambiental espacio temporal de la cuenca alta, enseguida se caracterizó las características físico - química y microbiológicamente de las aguas superficiales de la cuenca y se definieron los índices de calidad en sus distintos usos, así como la predicción de la dinámica de espacio temporal de los nutrientes utilizando SIG.

El Balsas avanza en dirección oeste por la parte norte del estado de Guerrero, un tramo en el que es llamado a veces río Mexcala. Recibe muchos afluentes no muy largos, de pequeños valles, como el río Amacuzac, que llega por la mano derecha desde Morelos. El valle del río es cruzado por el Puente Mezcala Solidaridad de la Autopista del Sol (95D) que comunica a la ciudad de Cuernavaca (en el estado de Morelos) con Acapulco, en la costa del estado de Guerrero. Después el río llega a la cola del embalse de la presa de El Caracol, donde recibe por la derecha, al río San Juan. Sigue hacia el este su discurrir incorporando nuevos afluentes, como los ríos Yextla y El Aguacate y atravesando varias localidades, como San Miguel Totolapan, Ajuchitlán del Progreso y Tlapehuala. Bordea por el sur la Ciudad Altamirano, casi en la frontera entre Guerrero y Michoacán, que con 25.317 hab. en 2010 es la ciudad más importante en el curso medio del río y tras atravesar Coyuca de Catalán recibe por la derecha al río Cutzamala, justo en la frontera interestatal. A partir de aquí y hasta su desembocadura, el Balsas forma la frontera entre los estados de Guerrero (al sur) y Michoacán (al norte). En este largo tramo incorpora las aguas de los afluentes Placeres del Oro y Chiquito y pasa por la pequeña localidad de Zirándaro de los Chávez. Llega después el río a la larga cola del embalse de la presa del Infiernillo, $\mathrm{y}$, en ese tramo embalsado, recibe al río Grande y al río Cupatitzio. Tras más de $70 \mathrm{~km}$ de tramo embalsado llega a la presa, en Infiernillo, y tras 
virar finalmente hacia el sur, sale de los valles montañosos interiores y emprende su último tramo, ya en la vertiente costera. Las estaciones son 1) Cuahulotitlan, 2) Altamirano, 3) Morelita, 4) Coyuca 5) San Miguel Totolapan, 6) Ajuchitlan, 7) Zirandaro, 8) El Progreso y 9) Cutzamala.

\section{MATERIALES Y MÉTODOS}

En el año 2018, en época de estiaje, se tomaron muestras simples en un día normal de operación industrial para determinar cuantitativa y cualitativamente la contaminación producida. Los parámetros estudiados se agruparon en cinco categorías: 1) De campo, tales como caudal del rio, materia flotante, color, olor, temperatura, CE, pH y OD; 2) Fisicoquímicos: 2A. sólidos suspendidos totales (SST), sólidos disueltos totales (SDT), sólidos sedimentables (SSe), demanda bioquímica de oxígeno (DBO5), demanda química de oxígeno (DQO), grasas y aceites (GyA); 2B. nutrientes: nitrógeno total (NTotal), nitrógeno amoniacal (Namoniacal), nitrógeno orgánico (Norg), nitrógeno como nitritos y nitratos (Nnitritos +Nnitratos), nitrógeno nitritos (Nnitritos), fósforo total (Ptotal), fósforo orgánico (Porg) y ortofosfatos (Porto); 3) Metales pesados (As, $\mathrm{Cd}, \mathrm{Cu}, \mathrm{Cr}$, $\mathrm{Cr}+6, \mathrm{Hg}, \mathrm{Ni}, \mathrm{Pb}, \mathrm{Zn}, \mathrm{Al}, \mathrm{Fe}$ y $\mathrm{Mn}$ ); 4) Microbiológicos (coliformes fecales) y 5) Toxicidad (Vibrio fischeri, Daphnia magna ) y Mutagenicidad (prueba de Ames) con dos cepas (TA98 y TA100), sin y con activación metabólica (-S9 y +S9). En las cuatro primeras categorías, para determinar la calidad del agua del río se tomó como referencia los límites máximos permisibles (LMP) establecidos en la NOM-001-ECOL-1996; así como los criterios ecológicos de calidad del agua (CE CCA-001/89) y La Ley Federal de Derechos de Agua (LFA, 2007). Los análisis de toxicidad y mutagenicidad se realizaron de acuerdo a las normas mexicanas (NMX-AA112-1995SCFI) y (NMX-AA087-1995-SCFI) y a la (OECD-471, 1995).

\section{RESULTADOS}

\section{Parámetros de campo}

Los valores obtenidos se presentan en la Tabla I. La estación con el máximo caudal es la 8 (5686 1·s-1), mientras que la estación 1 presenta el caudal más bajo (273 1·s-1). La materia flotante, presente sólo en la estación 4, fue de tipo doméstico (botellas PET, plásticos y latas); la norma NOM-001-ECOL-1996 establece ausencia de materia flotante. En el color son evidentes los cambios a lo largo de los nueve sitios, iniciando con un color café en las estaciones 1 y 2 , transformándose en azul en la estación 3, negro en las estaciones 4 a 8 para, finalmente terminar en gris en la estación 9. Las estaciones 1, 2 y 3 no presentan olor alguno, mientras que de la 4 a la 9 mostraron un olor a ácido sulfhídrico. La temperatura en el agua del río osciló entre 14 y $19^{\circ} \mathrm{C}$ entre las estaciones 1 y 9 ; los CE-CCA-001/89 establecen condiciones naturales de temperatura de más de $1,5^{\circ} \mathrm{C}$ Los valores mínimos de conductividad eléctrica se presentaron en las estaciones 1 y 2 (213 y $250 \mu \mathrm{S} \cdot \mathrm{cm}-2)$, y el valor máximo se registró en la estación $8(945 \mu \mathrm{S} \cdot \mathrm{cm}-2)$. Los $\mathrm{pH}$ de 8 estaciones son similares $(77,5)$, excepto la estación 3 con un $\mathrm{pH}$ de 8 . Para los parámetros de color, olor, $\mathrm{CE}$ y $\mathrm{pH}$ no se han establecido LMP en la NOM-001-ECOL-1996 ni en los CE-CCA-001/89. La concentración máxima de OD se registró en la estación 2 (8,6mg·1-1), seguida por las estaciones 1,7 y $6(6,4 ; 6,1$ y $5,9 \mathrm{mg} \cdot 1-1)$ y el valor mínimo (1,7mg.1-1) se localizó en la estación 9, encontrándose fuera de los CE-CCA-001/89. 
Tabla 1. Parámetros de campo

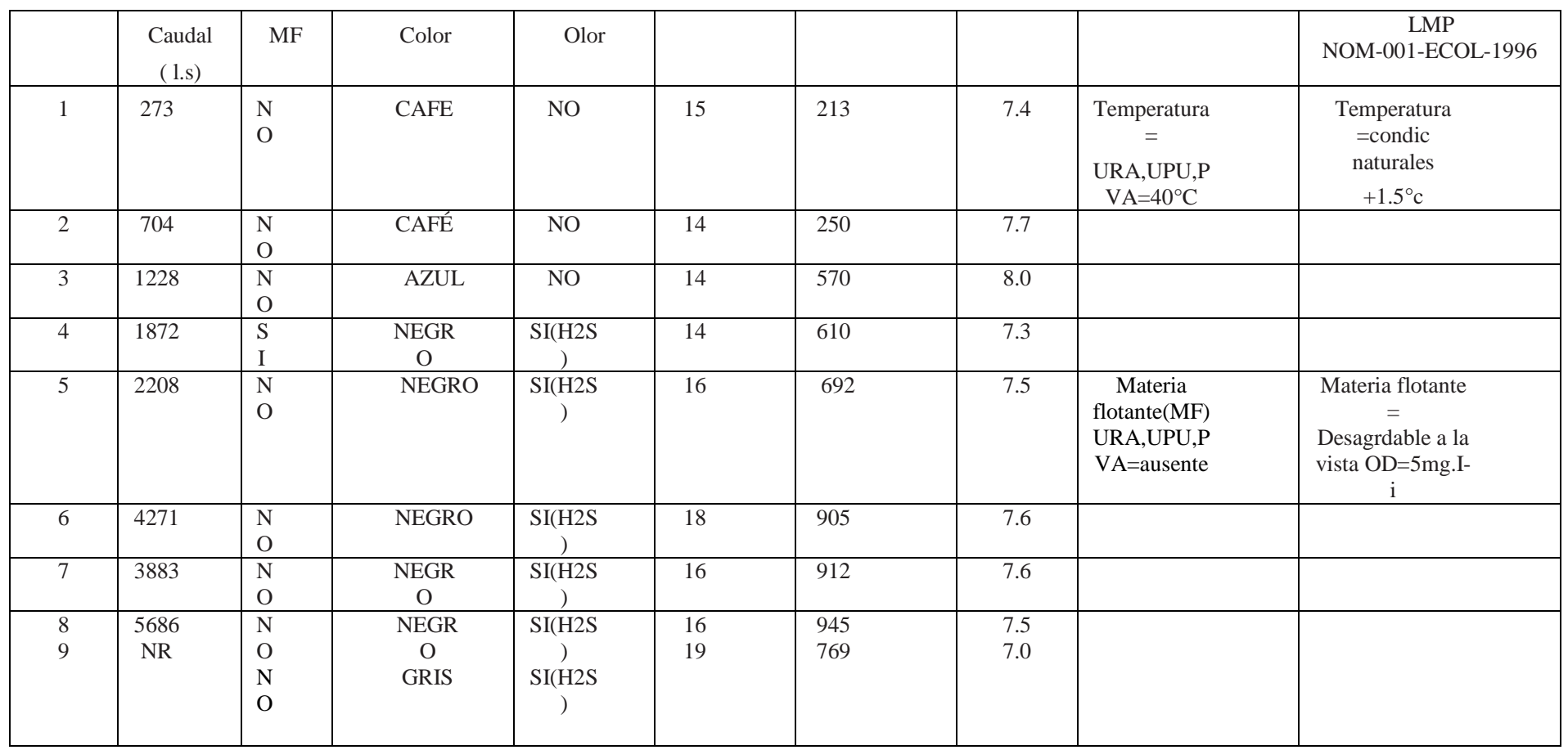

LMP: límite máximo permisible ,URA :uso riego agrícola , UPU :uso publico PVA: protección vida acuática NR: no realizado

Parámetros fisicoquímicos

En la Tabla II se muestran los valores de los parámetros SST, SDT, SSe, DBO5, DQO y GyA. El valor mínimo de SST se obtuvo en la estación 1 (8mg·1-1) y el máximo en la estación 7 (343mg·1-1). El LMP de SST para Uso de Riego Agrícola (URA) es rebasado en las estaciones 7, 8 y 9. En las estaciones 6 a 9 se sobrepasa el LMP para Uso Público Urbano (UPU), mientras que para Protección de Vida Acuática (PVA) el LMP es excedido en todas las estaciones. Solamente se registró SDT (11mg·1-1), parámetro que no se incluye en la normativa vigente, en la estación 7. Para SSe todas las estaciones cumplen con los LMP establecidos para URA, UPU y PVA. Las concentraciones mínima y máxima de DBO5 se registraron en las estaciones 1 y 7 (11 y 270mg.1-1), siendo rebasado el LMP para URA en las estaciones 5 y 9; para UPU en las estaciones 5, 6 y 7; y para PVA en las estaciones 3 (Morelita) a la 9 ( Cutzamala). (1841mg.1-1) en la estación 9, siendo rebasado el LMP establecido en la Ley Federal de Derechos en Materia de Agua en las estaciones
3 a 9 . El dato mínimo para GyA (15mg.1-1) fue en la estación 3 y el máximo (26mg-1-1) en las estaciones 5 y 8 . Estas dos últimas estaciones no cumplen con el LMP establecido en la NOM-001-ECOL-1996. En la Tabla III se muestran los valores obtenidos para $\mathrm{N}$ y $\mathrm{P}$ en sus diferentes formas. La concentración mínima de Ntotal detectada en las estaciones 1 y 8 fue de $2471 \mathrm{mg} \cdot 1-1$ y la máxima de 26551mg.1-1; ninguna de las estaciones cumple con los LMP para URA, UPU y PVA. El valor mínimo de Namoniacal registrado fue de 0,5mg·1-1 y el máximo El valor mínimo de DQO (22mg.1-1) se observó en la estación 1 y el máximo de 15400 en las estaciones 1 y 8 , respectivamente; sólo la estación 1 cumple con el LMP establecido en los CECCA-001/89. Los LMP para Norg y para Nnitritos+Nnitratos no se contemplan en la legislación actual. El valor para Nnitritos en todas las estaciones está dentro del LMP de los CE-CCA-001/89. En cuanto al Ptotal el valor mínimo y el máximo, de 1,0 y 6,0mg·1-1 en las estaciones 2 y 8 , cumplen con el LMP establecido en la NOM-001-ECOL-1996. Por 
otro lado, ninguna estación cumple con el LMP que marcan los CE-CCA-001/89. Se cuantificaron fosfatos orgánicos y ortofosfatos en todas las estaciones, aunque estos compuestos no se encuentran regulados por ninguna norma.

Tabla 2. Parámetros fisioquímicos tradicionales (ND: no detectado, NR: no realizado)

\begin{tabular}{|c|c|c|c|c|c|c|c|c|c|c|}
\hline Estación & $\begin{array}{c}\mathrm{N} \\
\text { total }\end{array}$ & $\begin{array}{c}\mathrm{N} \\
\text { amoniacal }\end{array}$ & $\begin{array}{c}\mathrm{N} \\
\text { org }\end{array}$ & $\begin{array}{c}\mathrm{N}- \\
\text { nitritos } \\
+\mathrm{N}- \\
\text { nitratos }\end{array}$ & $\begin{array}{l}\mathrm{N}- \\
\text { nitritos }\end{array}$ & $\begin{array}{c}P \\
\text { total }\end{array}$ & $\begin{array}{c}\mathbf{P} \\
\text { org }\end{array}$ & $\begin{array}{c}\mathbf{P} \\
\text { orto }\end{array}$ & $\begin{array}{c}\text { LMP } \\
\text { NOM-001- } \\
\text { ECOL-1996 } \\
\text { mg-1 }^{-1}\end{array}$ & $\begin{array}{c}\text { LMP } \\
\text { CE-CCA- } \\
\text { 001/89 } \\
\text { mg-1 }^{-1}\end{array}$ \\
\hline \multicolumn{11}{|c|}{${\mathrm{mg}-1^{-1}}^{-1}$} \\
\hline $\mathbf{1}$ & 2471 & 0.5 & 1 & 1 & 0.09 & 1 & 0.3 & 0.3 & & \\
\hline 2 & 4223 & 0.9 & 1778 & 1 & 0.07 & 1 & 0.0 & 0.3 & & \\
\hline 3 & 15772 & 6952 & 8448 & 0.4 & 0.02 & 2 & 0.4 & 1.2 & $\mathrm{~N}_{\text {total }}$ URA, & \\
\hline 4 & 1686 & 9746 & 6864 & 1.3 & 0.03 & 2 & 0.2 & 1.4 & UPU, PVA= & $\mathrm{N}_{\text {amoniacal }}=$ \\
\hline 5 & 16531 & 5654 & 10296 & 0.6 & 0.02 & 4 & 0.2 & 2.2 & $60,60,25$ & 0.6 \\
\hline 6 & 14480 & 8118 & 5962 & 0.4 & 0.02 & 3 & 0.8 & 2.0 & & \\
\hline 7 & 22420 & 11990 & 9900 & 0.5 & 0.03 & 5 & 0.5 & 2.9 & $P_{\text {total }}$ URA, & Nitritos $=0.6$ \\
\hline 8 & 26551 & 15400 & 10670 & 0.5 & 0.02 & 6 & 0.9 & 1.5 & UPU, PVA= & \\
\hline 9 & 11660 & 9339 & 2321 & NR & 0.02 & 2 & 0.06 & NR & $30,30,10$ & $\mathrm{P}_{\text {total }}=0.05$ \\
\hline
\end{tabular}

Metales

En la Tabla IV se muestran los metales cuantificados. Los valores de As y $\mathrm{Cd}$ se encuentran dentro del LMP de la NOM001ECOL-1996. Se detectó $\mathrm{Cr}+6$ en la estación 8 con una concentración $0,13 \mathrm{mg} \cdot 1-1$, rebasando significativamente el LMP establecido en los CE-CCA-001/89. La existencia de $\mathrm{Hg}$ en todas las estaciones muestra concentraciones de 0,001mg.l-1 y cumple con la NOM-001ECOL-1996, excepto en la estación $5(0,002 \mathrm{mg} \cdot 1-1)$ que rebasa el LMP de los CECCA-001/89. El Ni en todas las estaciones muestra concentraciones por debajo de la NOM-001-ECOL-1996 y no se le considera en los CE-CCA-001/89. El Fe y el Mn no se detectaron de la estación 1 a la 7 , pero el Fe está presente en las estaciones 8 y 9 (0,53 y $0,51 \mathrm{mg} \cdot 1-1)$, mientras que el $\mathrm{Mn}$ solo se detectó en la estación 9 (0,52mg.1-1); para ninguno de ellos hay LMP en la NOM-001ECOL-1996 ni en los CE-CCA-001/89.

Tabla 3. Parámetros relacionados con materia orgánica (Nr: no realizado).

\begin{tabular}{|c|c|c|c|c|c|c|c|c|c|}
\hline Estación & $\begin{array}{c}\text { SST } \\
\text { mg-1 }^{-}\end{array}$ & $\begin{array}{c}\text { SDT } \\
\text { mg-1 }^{-1}\end{array}$ & $\begin{array}{c}\text { SSc } \\
\text { m1- } \\
1^{-1}\end{array}$ & $\begin{array}{l}\mathrm{DBO}_{5} \\
\mathrm{mg-1}^{-1}\end{array}$ & $\begin{array}{l}\text { DQO } \\
\text { mg-1 }^{-1}\end{array}$ & $\begin{array}{c}\text { Grasas/aceites } \\
\text { mg-1 }^{-1}\end{array}$ & $\begin{array}{c}\text { LMP } \\
\text { NOM-001-ECOL-1996 }\end{array}$ & $\begin{array}{c}\text { LMP } \\
\text { CE-CCA-001/89 }\end{array}$ & $\begin{array}{l}\text { LMP } \\
\text { LFD- } \\
2008\end{array}$ \\
\hline 1 & 64 & $<0.18$ & 0.6 & 11 & 22 & ND & SST, URU, UPU, & \multirow{13}{*}{$\begin{array}{c}\text { Grasas y aceites }= \\
\text { ausentes }\end{array}$} & $\mathrm{DQO}=$ \\
\hline 2 & 70 & $<0.18$ & 0.0 & 23 & 36 & ND & $\mathrm{PVA}=$ & & $100 \mathrm{mg}-1^{-}$ \\
\hline 3 & 109 & $<0.18$ & 0.0 & 87 & 189 & 15 & $200,125,60 \mathrm{mg}-1^{-1}$ & & 1 \\
\hline 4 & 80 & $<0.18$ & 0.7 & 88 & 157 & 24 & & & \\
\hline 5 & 110 & $<0.18$ & 0.6 & 259 & 357 & 26 & Ssc, URA, UPU, PVA= & & \\
\hline 6 & 172 & $<0.18$ & 0.7 & 157 & 314 & 22 & $2 \mathrm{ml}-1^{-1}$ & & \\
\hline 7 & 343 & 11 & 2 & 270 & 383 & 24 & & & \\
\hline 8 & 240 & $<0.18$ & 1 & 87 & 200 & 26 & DBO, URA, UPU, & & \\
\hline \multirow[t]{5}{*}{9} & 240 & $<0.18$ & 1 & 78 & 1841 & NR & $\mathrm{PVA}=$ & & \\
\hline & & & & & & & 200,150 y $60 \mathrm{mg}-1^{-1}$ & & \\
\hline & & & & & & & Grasas y aceites & & \\
\hline & & & & & & & RA, UPU, PVA= 25mg- & & \\
\hline & & & & & & & $1^{-1}$ & & \\
\hline
\end{tabular}


Las concentraciones de $\mathrm{Cu}$, Crtotal, $\mathrm{Pb}, \mathrm{Zn}$ y $\mathrm{Al}$ detectadas estuvieron debajo del LMP de la NOM-001ECOL-1996 en todas las estaciones.

\section{Microbiológicos}

El valor mínimo y el máximo de coliformes fecales se registraron en las estaciones 2 y 7 (2,20E3 y 3,08E07NMP/100ml). Ninguna de las estaciones cumple con el LMP establecido en la NOM-001-ECOL-1996 (Tabla VI).

Toxicológicos

Para evaluar los resultados de toxicidad con V. fischeri y D. magna (Tabla VI) se empleó la clasificación de toxicidad de acuerdo con Bulich (1982) y para la prueba de Ames los valores de razón de mutagenicidad (RM; Mortenmals, 2000). En las estaciones 1 y 2 no se detectó toxicidad con V. fischeri; en las estaciones 3 y 4 existe toxicidad con 3 y 2 unidades de toxicidad (UT), de las estaciones 5 a la 9 se registró niveles muy tóxicos (2818UT) debido a la presencia de compuestos orgánicos. La estación 3 se mostró levemente tóxica con D. magna (1UT) y las estaciones 4 a 9 resultaron tóxicas (24UT), debido probablemente a la presencia de compuestos inorgánicos del tipo metales y metaloides.

Tabla 4. Metales pesados y metaloides

\begin{tabular}{|c|c|c|c|c|c|c|c|c|c|c|c|c|c|c|}
\hline ESTACIÓN & As & $\mathrm{Cd}$ & $\mathrm{Cu}$ & $\mathrm{Cr}$ & $\mathrm{Cr}^{16}$ & $\begin{array}{c}\mathrm{Hg} \\
\mathrm{mg}-1^{-1}\end{array}$ & $\mathrm{Ni}$ & $\mathrm{Pb}$ & $\begin{array}{l}\mathrm{Zn} \\
-\end{array}$ & $\mathrm{Al}$ & $\mathrm{Fe}$ & $\mathrm{Mi}$ & $\begin{array}{c}\text { I.MP } \\
\text { NOM-001-ECOL-1996 }\end{array}$ & $\begin{array}{c}\text { I.MP } \\
\text { CE-CCA- } \\
001 / 89\end{array}$ \\
\hline 1 & $<0,005$ & $<0,02$ & $<0,05$ & $<0,05$ & $\mathrm{ND}$ & 0,002 & $<0,05$ & $<0,10$ & $<0,1$ & $<5,0$ & $\mathrm{ND}$ & ND & $\begin{array}{c}m g-1^{-1} \\
\text { As URA. UPU, PVA=0,2: } \\
0,1: 0,1\end{array}$ & $\begin{array}{c}\mathrm{mg}-1^{-1} \\
\text { As III }=0,2\end{array}$ \\
\hline 2 & $<0,005$ & $<0,02$ & $<0,05$ & $<0,05$ & ND & 0,001 & 0,017 & $<0,10$ & $<0,1$ & $<5,0$ & ND & ND & $\begin{array}{l}\text { Cd URA, UPU, PVA= 0,2: } \\
01: 0,1\end{array}$ & \\
\hline 3 & $<0,005$ & $<0,02$ & $<0,05$ & $<0,05$ & $\mathrm{ND}$ & 0,001 & $<0,05$ & $<0,10$ & $<0,1$ & $<5,0$ & ND & ND & $\begin{array}{c}\mathrm{Cu} \text { URA, UPU, PVA=4,0; } \\
4,0 ; 4,0\end{array}$ & $\mathrm{Cn}=0,0005$ \\
\hline 4 & $<0,005$ & $<0,02$ & $<0,05$ & $<0,05$ & $\mathrm{ND}$ & 0,001 & $<0,05$ & $<0,10$ & $<0,1$ & $<5,0$ & ND & ND & $\begin{array}{c}\text { Cr URA, UPU, PVA= }=1,0 \\
0,5: 0,5\end{array}$ & \\
\hline 5 & $<0,005$ & 0,04 & $<0,05$ & $<0,05$ & $\mathrm{ND}$ & 0.002 & $<0,05$ & $<0,10$ & $<0,1$ & $<5,0$ & ND & ND & $\begin{array}{c}\text { Hg URA, UPU UPVA= } \\
0,01 ; 0,005 ; 0,005\end{array}$ & $\mathrm{Cr}^{16}=0,01$ \\
\hline 6 & $<0,005$ & $<0,02$ & $<0,05$ & $<0,05$ & ND & 0,001 & 0,017 & $<0,10$ & $<0,1$ & $<5,0$ & ND & ND & $\begin{array}{c}\text { Ni URA, UPU, PVA=0,5; } \\
0,2: 0,2\end{array}$ & \\
\hline 7 & 0,01 & 0,05 & $<0,05$ & $<0,05$ & $\mathrm{ND}$ & 0.001 & $<0,05$ & $<0,10$ & $<0,1$ & $<5,0$ & ND & ND & $\begin{array}{c}\mathrm{Pb} \text { URA, UPU PVA= }=0,2 ; \\
0,1 ; 0,1\end{array}$ & $\mathrm{Hg}=0,001$ \\
\hline 8 & $<0,005$ & $<0,02$ & $<0,05$ & $<0,05$ & 0,13 & $<0,0005$ & $<0,05$ & $<0,10$ & $<0,1$ & $<5,0$ & 0,53 & ND & $\begin{array}{c}\text { Zn URA, UPU, PVA= } 10,0 ; \\
10,0 ; 10,0\end{array}$ & \\
\hline 9 & $<0,005$ & $<0,02$ & $<0,05$ & $<0,05$ & ND & $<0,0005$ & $<0,05$ & $<0,10$ & $<0,1$ & $<5,0$ & 0,51 & 0,52 & & \\
\hline
\end{tabular}


Tabla 5. Parámetros microbiologicos, toxicologicos y mutegenicos.

\begin{tabular}{|c|c|c|c|c|c|c|c|c|c|c|}
\hline ESTACIÓN & $\begin{array}{c}\text { Colifornes } \\
\text { fecales } \\
\text { NMP/100m } \\
1\end{array}$ & $\begin{array}{l}\text { Vibrio fischeri } \\
\mathrm{CE}_{50}(\%) \\
-\overline{\mathrm{UT}}\end{array}$ & $\begin{array}{c}\text { Daphnia } \mathrm{CE}_{50} \\
-\mathrm{UT}\end{array}$ & $\begin{array}{c}\text { mag } \\
\text { na } \\
(\%)\end{array}$ & $\begin{array}{c}\text { CEP } \\
\mathrm{A} \\
\underline{-S 9} \\
\end{array}$ & $\begin{array}{c}\text { TA9 } \\
8 \\
+\mathrm{S} 9 \\
\end{array}$ & $\begin{array}{l}\text { CEPA } \\
\underline{-S 9}\end{array}$ & $\begin{array}{l}\text { TA100 } \\
+ \text { S9 }\end{array}$ & $\begin{array}{c}\text { LMP } \\
\text { (NOM-001-ECOL- } \\
1996)\end{array}$ & $\begin{array}{l}\text { Clasif. De toxicidad } \\
\text { (Bulich, 1982) }\end{array}$ \\
\hline 1 & $2,64 \mathrm{E} 03$ & TND & TND & TND & TND & - & - & - & Coliformes fecales & $>4$ Muy toxica \\
\hline 2 & 2,20E03 & TND & TND & TND & TND & - & - & - & $\begin{array}{c}\text { URA }= \\
1000 \mathrm{NMP} / 100 \mathrm{ml}\end{array}$ & 2-4 Toxica \\
\hline 3 & 2,64E06 & 33 & 3 & 82 & 1 & 6 & - & - & & $\begin{array}{c}1.33-1,99 \\
\text { Moderadamente tox. }\end{array}$ \\
\hline 4 & 2,64E06 & 40 & 2 & 53 & 2 & 5 & - & - & & $\begin{array}{c}<1,33 \text { Levemente } \\
\text { toxica }\end{array}$ \\
\hline 5 & 2,53E06 & 4 & 28 & 57 & 2 & 7 & - & - & I.MP & $\begin{array}{c}\text { Clasif. De } \\
\text { mutagenecidad }\end{array}$ \\
\hline 6 & 2,64E06 & 17 & 6 & 63 & 2 & 63 & - & - & (CE-CCA-001/89) & $\begin{array}{c}\text { (mortenmals y Zeiger, } \\
2000)\end{array}$ \\
\hline 7 & $3,08 \mathrm{E} 07$ & 18 & 6 & 52 & 2 & - & - & - & & $<2=$ negativo \\
\hline 8 & 2,53E06 & 5 & 19 & 24 & 4 & 13 & 5 & - & $\begin{array}{c}\text { Colif. Fec. } \\
<200 \mathrm{NMP} / 100 \mathrm{ml}\end{array}$ & $>2=$ positivo \\
\hline 9 & $2,64 \mathrm{E} 07$ & 6 & 18 & 32 & 3 & 14 & 5 & - & & \\
\hline
\end{tabular}

Prueba de Ames. En las estaciones 1, 2 y 7 no se detectó mutagenicidad (ND) con la cepa TA98 sin activación metabólica, pero sí en las estaciones $3,4,5,6,8$ y 9 con valores de 6,5 , $7,63,13$ y 14RM respectivamente, siendo significativamente más alta la estación 6. Con la cepa TA98 con activación metabólica no se registró mutagenicidad en las estaciones 1 a 7 , pero sí se registró en las estaciones 8 y 9 con valores de 5RM (Tabla V). Empleando la cepa TA100 sin activación metabólica, en las estaciones 1 a 6 no se detectó mutagenicidad, pero las estaciones 7,8 y 9 presentan valores mutagénicos de 5, 7 y 6RM. Empleando la misma cepa con activación metabólica no se detectó mutagenicidad en ninguna de las estaciones (Tabla 5).

\section{DISCUSIÓNES Y CONCLUSIONES}

El caudal del río Balsas se incrementa desde la primera medición en la estación 1 hasta la estación 6 en 15 veces, de 273 a $42711 \cdot s-1$, disminuyendo en la estación 7 a 38831·s-1. En todo el afluente de donde nace hasta el cauce en la región Calentana podemos encontrar industrias, agricultura, ganadería, minerías, basureros, drenajes, etc.; lo cual conlleva extracción de agua del río para sus procesos. El factor determinante para esta clasificación fue la cantidad de parámetros que sobrepasan los LMP de la legislación). La disminución del caudal en la estación 7 muestra la necesidad de limitar la extracción del recurso hídrico. Los parámetros fisicoquímicos fueron rebasados en todas las estaciones en el siguiente orden 1 $<2<4,6<3,5,9<8<7$. Las estaciones 7 y 8 (Tablas 3 y 4) se destacan por los máximas valores de SST, SDT, SSe, DBO5, DQO, GyA, Ntotal, Namoniacal, Norg, Ptotal y Porg, mostrando el impacto de las descargas de origen industrial y municipal en el río, confirmando lo reportado por Valladares et al., (2005). Los metales detectados de menor a mayor concentración fueron: $\mathrm{Hg}$ (en estación 5) $<\mathrm{As}($ en 7$)<\mathrm{Ni}($ en 7$)<\mathrm{Cd}($ en 7$)<\mathrm{Cr} 6+$ (en 8) $<\mathrm{Mn}$ (en 9) $<\mathrm{Fe}$ (en estación 8). La presencia de estos metales concuerda con los resultados de otros estudios concuerda (Silva et al., 2002; Méndez et al., 1995; Saldaña y Gómez, 2006; CONAGUA, 2007b; Navarro et al., 2007) El cambio de color en las estaciones 1 y 2 fue influenciado por la fuerza de la 
corriente, que arrastra partículas suspendidas que modifican el color natural; en la estación 3 el cambio se debe a la descarga textilera del teñido de telas, específicamente mezclilla. Los cambios subsecuentes se deben a las descargas de industrias procesadoras de alimentos, metalúrgicas, químicas y de colectores industriales, que imparten al agua un color negro de las estaciones 4 a 8 . Estas descargas provocan también el surgimiento de olor a ácido sulfhídrico a partir de la estación 4, indicando aumento en la actividad biológica y contaminación industrial severa. La variación de temperatura de la estación 1 a la 9 fue de $4^{\circ} \mathrm{C}$, aumento debido, probablemente, a procesos industriales que generan agua caliente que influye en la tasa de crecimiento biológico, en las reacciones químicas, en la solubilidad de los contaminantes y en el desarrollo de la biota. La conductividad eléctrica en la estación 3, donde inician las descargas industriales, se incrementa significativamente hasta la estación 8 disminuyendo en la 9. A mayor conductividad eléctrica, mayor es el contenido de sólidos disueltos en formas iónicas y provenientes de las descargas industriales. El valor de $\mathrm{pH} 8$ registrado en la estación 3 se debe a las descargas municipales y a la actividad de procesos industriales (textil, químico $\mathrm{y}$ petroquímico). Algunas industrias utilizan procesos neutralizantes en sus descargas a base de bicarbonatos y carbonatos que justificarían el incremento del $\mathrm{pH}$, específicamente en esta estación, pero ello no puede asegurarse. La estación 2 recibe descargas municipales, no obstante, tiene el nivel de OD más alto, indicando que posee capacidad para mantener vivos organismos aerobios y plantas. Las otras estaciones muestran niveles de OD dentro del límite establecido en los CE-CCA-001/89, por lo que el río cuenta con capacidad autodepurativa (Sperling, 2001). Sólo el valor de OD en la estación 9 está fuera de la norma, indicando contaminación en ese punto e imposibilitando la supervivencia de la biota (Iriondo y Mota, 2004). Los parámetros de campo muestran el estado tangible del río, las variaciones en el caudal y su velocidad, los cambios de color y olor muestran la magnitud de las descargas que lo impactan, causantes de su deterioro. $\mathrm{La}$ estación 7 sobresale por sus valores altos de SST y SDT debido a partículas orgánicas (materia descompuesta y compuestos químicos) e inorgánicas (iones y moléculas disueltos en el agua, minerales, metales) en solución, que podrían interactuar con los agentes químicos y biológicos responsables de los cambios de color (negro) y olor (ácido sulfhídrico) antes discutidos, que pueden incrementar la toxicidad. La estación 7 también resalta por sus altos niveles de DBO5 y DQO (Guerra-Hernández et al., 2008), lo cual indica la insuficiencia de $\mathrm{O} 2$ necesario para degradar por medios biológicos y oxidar la materia orgánica por medios químicos (Sperling, 2001). La estación 9 muestra también un DQO alto debido a que es el reservorio de toda la materia orgánica que se vierte al río. Las estaciones 5 y 8 muestran valores de GyA elevados, debido a los procesos que se llevan a cabo en las industrias química, petroquímica, textil y farmacéutica, y descargas municipales. La grasa en aguas residuales interfiere con la vida acuática creando películas de materiales en flotación y afectando los procesos biológicos naturales del agua. El Ntotal (Norgánico y Namoniacal) está presente en exceso en todas las estaciones, debido a desechos domésticos, agrícolas e industriales. El Namoniacal está presente en el agua en forma de amonia libre (NH3) y del catión amonio (NH4+), indicando contaminación reciente en el cuerpo de agua y su efecto es nocivo para la fauna acuática (Jiménez, 2002). Las estaciones 1, 2 y 4 presentan alta concentración de 
Nnitritos+Nnitratos. Es probable que la presencia de bacterias inicie un proceso de desnitrificación llegando hasta la generación de N2 molecular. Otras fuentes importantes de nitrato son los fertilizantes, principalmente en época de lluvia (Jiménez, 2002). La presencia de Ptotal en todas las estaciones se debe al uso excesivo de fertilizantes, detergentes y a procesos de descargas industriales. Los Porg y los Porto son producto de las descargas de agua de acondicionamiento para tuberías, torres de enfriamiento, calentadores y calderas. Siendo considerados como parámetros críticos en la calidad del agua por su influencia en el proceso de eutrofización, no se encuentran regulados por ninguna norma. Los resultados obtenidos muestran los metales que se encuentran por debajo del límite de detección del equipo a niveles traza; éstos son $\mathrm{Cu}, \mathrm{Cr}, \mathrm{Pb}, \mathrm{Zn}$ y $\mathrm{Al}$, no representando peligro en el río. As, Cd, Ni, Fe y Mn están por debajo de los LMP de la normativa mencionada; sin embargo, su afluencia debe monitorearse continuamente para detectar su origen. La presencia de As, Cd y Ni se debe a descargas de empresas ubicadas en la estación 7, principalmente de tipo metalúrgica, química, automotriz y al uso de pesticidas, de acuerdo con Klaassen y Watkins (2001). El Cd puede ser generado por industrias de pinturas, plásticos, y los lodos de aguas domésticas y/o tratadas. $\mathrm{El} \mathrm{Ni}$ presente en el río quizás proceda de plantas alimenticias, bebidas, tenería y textil. También se cuantificaron metales que no se incluyen en norma alguna, como Fe y $\mathrm{Mn}$. El primero solo se detectó en la estación 8, y ambos en la 9. Estos elementos se combinan con la materia orgánica formando coloides de difícil sedimentación que aportan un olor desagradable y color indeseable, lo cual coincide con Helmut, 1991; Rainer, 1991. Por arriba de los LMP establecidos los metales detectados fueron $\mathrm{Hg}$ y $\mathrm{Cr}+6$, en la estaciones 5 y 8 respectivamente. La presencia de $\mathrm{Hg}$ se debe a descargas de la industria textil, química, petroquímica y metalúrgica; no obstante no es posible asegurar que estas empresas hagan uso excesivo de $\mathrm{Hg}$ y/o sus derivados, lo que coincide con Klaassen y Watkins, 2001 y que sean responsables de su presencia en el río. La alta concentración de $\mathrm{Hg}$ puede derivar de cenizas dispersadas de volcanes (Popocatépetl) en el resto de las estaciones. La presencia de $\mathrm{Cr}+6$ en la estación 9 puede deberse a descargas de tipo textil y otras como tenerías, galvanoplastias, metalúrgicas, producción de pigmentos y fertilizantes. El exceso de coliformes y la DBO5 en la estación 7 tienen un origen común de contaminación, que puede ser identificado en la Figura 2 con descargas de tipo municipal e industrial (alimenticia, bebidas, tenerías, petroquímica, química y otras). Los hallazgos con V. fischeri muestran toxicidad moderada en las estaciones 3 y 4 , pero alta de la 5 a la 9 , posiblemente debido a las concentraciones elevadas de Ntotal, quizá como resultado de la descomposición de la materia orgánica. El Namoniacal forma NH3 y NH4+ en el agua, elevando la toxicidad. La toxicidad también puede deberse a la presencia de compuestos orgánicos, como hidrocarburos poliaromáticos, herbicidas y pesticidas. Con D. magna se detectó toxicidad leve en la estación 3 (1UT); en las estaciones 4 a 9 se detectaron valores de 2 a 4 UT, a pesar de que se registraron concentraciones traza de metales (Tabla VI). La prueba de Ames utiliza dos cepas, TA98 y TA100. La diferencia entre ellas es que la primera tiene una mutación frameshift y la otra una puntual en un par de bases (Takiya et al., 2003). Si las pruebas resultan positivas, existe un $99 \%$ de probabilidad de afectar la salud humana por carcinogénesis (Umbuzeiro et al., 2001; Takiya et al., 2003). Con la cepa TA98 -S9 se detectó mutagenicidad alta en la estación 6, mientras con TA98 +S9 en la estación 8 . Por 
otro lado, con la cepa TA100 -S9 se registró mutagenicidad alta en la estación 8 , pero no se detectó con la TA100 +S9. Los resultados de la prueba de Ames con S. typhimurium (TA98 y TA100 sin y con activación metabólica) sugieren la presencia de compuestos orgánicos (hidrocarburos poliarómaticos, pesticidas, emulsificantes, plastificantes, dispersantes) y compuestos volátiles orgánicos (benceno, tolueno, etc.), cuya presencia se deberá determinar en el río, lo cual coincide con Umbuzeiro et al., 2001; Takiya et al., 2003).

Los parámetros de campo indican el daño en el Río es causado por la contaminación debida a los asentamientos humanos y al desarrollo industrial al efectuar sus descargas en este cuerpo de agua, a pesar de haber dado un tratamiento previo a las mismas, lo cual resalta la necesidad de incluir pruebas de toxicidad y mutagenicidad en la legislación vigente. El grado de deterioro detectado con los parámetros de campo en las estaciones fue 1,2 $<3,5<4,6,7<8,9$.. La contaminación microbiológica rebasa el LMP en todas las estaciones, destacándose la 7 y la 9 (Tabla VII), resultados que coinciden con los de Silva et al., (2002) quienes reportaron contaminación del río por drenajes municipales e industriales. Los resultados de los análisis toxicológicos y mutagénicos revelaron la presencia de compuestos tóxicos que no fueron detectados por los métodos de análisis convencionales de la estación 3 a la 9 . V. fischeri registró toxicidad de menor a mayor concentración en las estaciones $4<3<7$ $<6<9<8<5$. D. magna, en orden de menor a mayor concentración, en las estaciones $3<6$ $<5<4<7<9<8$. La prueba de Ames detectó mutagenicidad de menor a mayor concentración en las estaciones $4<3<5<8<9$ $<6$. Los resultados posiblemente se deban a la presencia de compuestos orgánicos (HPA's), que no fueron determinados en este trabajo por no estar incluidos en las normativas actuales y están siendo evaluados a raíz de los resultados toxicológicos y mutagénicos. La norma vigente se respeta y se pone en práctica; sin embargo, no es suficiente para determinar con mayor precisión el deterioro en la calidad del agua del río. En la medida en que este tipo de bioensayos sea accesible será una alternativa para un mejor monitoreo de las descargas, por lo cual se hace necesario cambiar la legislación vigente como un reto a corto plazo, con el fin de generar información para la toma de decisiones por las instancias pertinentes, en concordancia con Saldaña et al. (2002a, b) y CONAGUA (2007a, b). Por otro lado, se debe mejorar la eficiencia de las PTARs municipales e industriales, ya que esto permitiría contar con medidas de saneamiento especificas para los problemas detectados en este estudio, tales como el implemento de tratamientos avanzados, con nuevas tecnologías de clarificación biológica con enzimas u organismos inmovilizados (Salgado et al., 2006; Núñez et al., 2007); de desodorización, empleando reactores biológicos como biofiltros (Le Cloire et al., 2001); de nanofiltración avanzada para eliminar SST (Adham et al., 2006); de remoción de nutrientes por procesos de nitrificación-desnitrificación, remoción de amonio acuoso (Arriaga et al., 2003), desgasificación del amoniaco, precipitación química, asimilación; de remoción de compuestos inorgánicos disueltos mediante electrodiálisis, intercambio iónico, ósmosis inversa y precipitación (Kabra et al., 2004); eliminación de coliformes mediante radiación solar (Rizzo, 2009); y remoción de compuestos orgánicos disueltos por adsorción u oxidación química (Bubnov et al., 2006).

\section{AGRADECIMIENTOS}

Al Instituto Tecnológico de Cd. Altamirano por la oportunidad de realizar este trabajo de investigación. 
LITERATURA CITADA

Adham S, Chiue K, Lehman G, Mysore C, Clouet J (2006) Optimization of Membrane Treatment for Direct and Clarified Water Filtration. Report Series 91111F ISBN 9781843399674. AWWARF. Denver, CO, EEUU. 196 $\mathrm{pp}$.

Arriaga ARE, Garcia LRA, Soto GGE (2003) A Peach stone activated carbon chemically modified to absorb aqueous ammonia. J. Chil. Chem. Soc. 48: 65-68. https://doi.org/10.4067/S0717-97072003

Bubnov AG, Grinevich VI, Maslova ON (2006) Barrierdischarge plasma treatment of surface water to remove organic compounds. Rus. J. Appl. Chem. 79: 934-940. https://doi.org/10.1134/S107042720606 Bulich AA (1982) A practical and reliable method for monitoring the toxicity of aquatic samples. Proc. Biochem. (March-April); 45-47.

CONAGUA (2007a) Estudio y Declaratoria de Clasificación de los Ríos Atoyac y Zahuapan. www.ccbalsas.org. $\mathrm{mx} /$ GruposConsejo/GrupoSegEvaluacio $\mathrm{n} /$

Presentaciones/ pres31sesion/6APresentacion_ RioAtoyac-Zahuapan.pdf

CONAGUA (2007b) Clasificación de Cuerpos de Agua Nacionales Río Atoyac y Zahuapan.www2.ine.gob.mx/ argas/pres_proname_ago2008_e_gutierr ez. Pdf

Flores QR, Aceves-Capri S, Ramírez MI (2004) Viabilidad del Potencial Uso Acuícola de la Carpa Común (Cyprinus carpio) en la Presa Manuel Ávila Camacho, Puebla, México. www.tupublicas.com/ docs/04-03-200453-expocien.pdf.

Guerra-Hernández E, Balán-Hernández LL, Guerrero OR, Campos LLS (2008) Una mirada a los recursos hídricos en la zona alta de la subcuenca del Río Atoyac.
Escobar et al., 2019

Mem. VII Cong. Int., XIII Cong. Nac., III Reg. de Ciencias Ambientales. 4-6 Junio. Cd Obregón, México.

Helmut AH (1991) Iron. En Merian E (Ed.) Metals and their Compounds in the Environment. VCH. Nueva York, NY, EEUU. pp 945-958.

INEGI (2004) Anuario Estadístico. Puebla. Tomo I y II. Instituto Nacional de Estadística, Geografía e Informática. Aguascalientes, México.

INEGI (2008). negi.org.mx/geografía/español/estados/ endomex/

sombreado_riocfm?c $=444 \& \mathrm{e}=18$

Iriondo A, Mota J (2004) Desarrollo de una red neuronal para estimar el oxígeno disuelto en el agua a partir de instrumentación de EDAR. XXV Jornadas de Automática. 8-10 de Septiembre. Universidad de Castilla la Mancha. Ciudad Real, España.

Jiménez CBE (2002) La contaminación ambiental en México. Cap. 2. La Contaminación del Agua. Limusa. México. pp. 33-315.

Kabra K, Chaudhary R, Sawhney RL (2004) Treatment of hazardous organic and inorganic compounds through aqueousphase photocatalysis: A review. Indust. Eng. Chem. Res. 43: 7683-7696. https://doi.org/10.1021/ie0498551

Klaasen CD, Watkins BJ (2001) Efectos tóxicos de metales. En Manual de Toxicología. Capítulo 23. McGrawHill. México. pp. 659-722.

Le Cloire P, Humeau P, Ramírez-López EM (2001) Biotreatments of odours: control and performance of biofilters and a bioscrubber. Water Sci. Technol. 44: 219-226.

https://oi.org/10.2166/wst.2001.0544

Ley Federal de Derechos de Agua (2007)

Reforma

www.diputados.gob.mx/Leyes Biblio/ ref/lfd/LFD_ref35_24dic07.pdf . 
Escobar et al., 2019

Mangas RE, Sánchez MM, Molina A, Hugo R, García-Flores LM, Muñoz-Gutiérrez I, Zumaquero RJL (2005) Análisis de las Políticas de Rehabilitación de Presas: El Caso de la "Presa de Valsequillo" en el Estado de Puebla. www. comunicacion.buap.mx/ ortajes_especiales/reportaje_especial_v alsequillo.html

Méndez GT, Flores RA, Palacios MS (1995) Presencia de $\mathrm{Pb}, \mathrm{Cr}$, Co y $\mathrm{Cd}$ en suelos regados con aguas residuales en el distrito de riego 030 Tecamachalco Estado de Puebla. Mem. XXVI Cong. Nac. Ciencia del Suelo. Cd. Victoria, México. p. 36.

Méndez GT, Rodríguez DL, Palacios M (2000) Impacto del riego con aguas contaminadas evaluadas a través de la presencia de metales pesados en suelos. Terra 18: 277-278.

Mortenmals K, Zeiger E (2000) The Ames Salmonella/microsome mutagenicity assay. Mut. Res. 455: 29-60. https://doi.org/10.1016/S0027-5107(00) Navarro AE, Rincón C, Rosas E, Aguilar AP, Contreras S, Caso LR, Bayona JM, Velasco F, Sánchez A, Vázquez A, Martínez CT, Díaz M, Pérez A, Malajevich A, Cuellar I, Navarrete D, Cid S (2007) El agua en la Subcuenca del Nexapa, México: Necesidad de una gestión integral. Conferencia Latinoamericana de Saneamiento. 12-16 Noviembre. Cali, Colombia. Norma Mexicana NMXAA-003-1980 SCFI Aguas Residuales-Muestreo www. semarnat.

gob.mx/leyesynormas/Normas\%20Mex icanas\%20vigentes/NMXAA-0031980.pdf.

Norma Mexicana NMX-AA087-1995-SCFI Análisis de Agua-Evaluación de Toxicidad Aguda con Daphnia magna Status (CrustaceaCladocero)-Método de
Prueba. www. semarnat.gob.mx/ leyesynormas/Normas $\% 20$

Mexicanas\%20vigentes/ NMX-AA087-1995.pdf.

Norma Mexicana NMX-AA-1121995-SCFI Análisis de Agua y SedimentosEvaluación de Toxicidad Aguda con Photobacterium phosphoreumMétodo de Prueba.www. .mx/leyesynormas/Normas\%20Mexican as\%20vigentes/NMXAA-112-1995.pdf.

Norma Oficial Mexicana NOM001-ECOL1996 Que Establece los Límites Máximos Permisibles de Contaminantes en las Descargas de Aguas Residuales en Aguas y Bienes Nacionales. www. economia.gob.mx/work/normas/noms/1 997/001-ecol. Pdf.

Núñez APN, Cruz SR, Folch MJL, Villegas E (2007) Degradación de los colorantes, remazol brilliant blue $\mathrm{R}$ y 4 phenylazoaniline con lacasas fúngicas libres e inmovilizadas. Mem. VII Cong. Nac. Biología Molecular y Celular de Hongos de la Sociedad Mexicana de Bioquímica. 5-8 Nov. Guanajuato, México.

OECD (1995) Guidelines for testing of chemicals Genetic Toxicology: Salmonella typhimurium.Reverse. Mutat. Assay 471: 1-9.

Rainer SE (1991) Manganeso. En Merian E (Ed.) Metals and their Compounds in the Environment. VCH. Nueva York, NY, EEUU. Cap. II-19.

Rizzo L (2009) Inactivation and injury of total coliform bacteria after primary disinfection of drinking water by $\mathrm{TiO} 2$ photocatalysis. J. Haz. Mat. 164: 48-51.https://doi.org/10.1016/j.jhazmat. 2008.09.068

Saldaña FP, Gómez BMA (2006) Caracterización de fuentes puntuales de contaminación en el río Atoyac, México. Mem. XXX Cong. Interam. Ingeniería 
Sanitaria y Ambiental. 26-30 Nov.

Punta del Este, Uruguay.

Saldaña FP, Alcocer Y VH, Lerdo de Tejada

BA, Gómez BMA (2002a) Calidad del

Agua en Colectores de la Ciudad de Puebla y la Aplicación de Análisis de Toxicidad. XXVIII Cong. Interam. Ingeniería Sanitaria y Ambiental. 27-31 Oct. Cancún, México.

Saldaña, P, Lerdo de Tejada A, Gómez MA, López R (2002b) La importancia de incluir análisis de toxicidad en descargas industriales y municipales que afectan los cuerpos receptores. Cong. Nac. Ingeniería Sanitaria y Ciencias Ambientales. 17-20 Abr. Guanajuato, México.

Salgado J, Sandoval AM, Espinosa E, Dantán E, Silva S, Méndez M, Folch JL, Villegas E (2006) Purificación y caracterización de lacasas extracelulares de Pycnoporus sanguineus aplicadas al tratamiento de colorantes y evaluación toxicológica del proceso. Mem. V Cong. Int. y XI Cong. Nac. de Ciencias Ambientales. 7-9 de Junio. Oaxtepec, México.

Secretaría de Desarrollo Urbano y Ecología (SEDUE) (1989) Acuerdo por el que se Establecen los Criterios Ecológicos de Calidad de Agua CE-CCA-001/1989.
Diario Oficial de la Federación del 2/12/1989. Tomo CDXXX, No 9. México.

Silva GSE, Muñoz OA, De la Isla de Bauer ML, Infante GS (2002) Contaminación ambiental en la región de Atlixco: 1 Agua. Terra 20: 243-251.

Sperling MV (2001) Principios Básicos do Tratamiento de Esgotos. $4^{\mathrm{a}}$ ed. Universidade Federal de Minas Gerais. Brasil. 211 pp.

Takiya T, Horie Y, Futo S, Matsumoto Y, Kawai K, Zuzuki T (2003) Rapid selection of nonhotspot mutants among hidD+ revertants of Salmonella typhimurium TA98 in Ames test by peptide nucleic acid (PNA)-mediated PCR clamping. J. Biosci. Bioeng. 96: 588-590. https://doi.org/10.1016/S138-1 723(04)70156-5

Umbuzeiro A, Roubicek D, Sánchez P, Sato MZ (2001) The Salmonella mutagenicity assay in a surface water quality monitoring program based on a 20-year survey. Mut. Res. 49: 119-126. https://doi.org/10.1016/S1383-5718(01)

Valladares MR, Flores E, Navarro I (2005) Estudio de la calidad del agua residual que descarga al Río Atoyac: comunidades, ong, e investigadores unidos. II Encuentro participación de la mujer en la Ciencia. 19-20 Mayo. León, México. 
Copyright (c) 2019 Ludybed Escobar Sarabia, Maria Guadalupe Álvarez Diaz, Diana Pérez de Jesús y Francisco Zavala Hernández

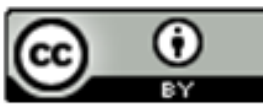

Este texto está protegido por una licencia licencia CreativeCommons 4.0.

Usted es libre para Compartir — copiar y redistribuir el $\mathrm{m}$ aterial en cualquier medio o formato- y Adaptar el documento —remezclar, transformar y crear a partir del material- para cualquier propósito, incluso p ara fines com erciales, siempre que cumpla la condición de:

Atribución: Usted debe dar crédito a la obra original de manera adecuada, proporcionar un enlace a la licencia, e in dicar si se han realizado cambios. Puede hacerlo en cualquier forma razonable, pero no de forma tal que sugiera que tiene el apoyo del licenciante o lo recibe por el uso que hace de la obra.

$\underline{\text { Resumendelicencia }}$ - Textocompletodelalicencia 